\title{
Evaluation of a polysaccharide conjugate vaccine to reduce colonization by Campylobacter jejuni in broiler chickens
}

Douglas C Hodgins ${ }^{1 \dagger}$, Neda Barjesteh ${ }^{1 \dagger}$, Michael St. Paul ${ }^{1,3}$, Zuchao Ma ${ }^{2}$, Mario A Monteiro ${ }^{2}$ and Shayan Sharif $f^{*}$

\begin{abstract}
Background: Campylobacter jejuni is a leading bacterial cause of food-borne illness in humans. Symptoms range from mild gastroenteritis to dysentery. Contaminated chicken meat is the most common cause of infection. Broiler chickens become colonized with high numbers of C. jejuni in the intestinal tract, but do not become clinically ill. Vaccination of broiler chicks to control colonization by C. jejuni is challenging because immune function is limited in the first 2 weeks post-hatch and immune suppressive maternal antibodies are common. In addition, there is little time for induction of immunity, since broilers reach slaughter weight by 5- 6 weeks of age. In the current study the immunogenicity of a C. jejuni capsular polysaccharide - diphtheria toxoid conjugated vaccine (CPSConj), administered subcutaneously with various adjuvants was assessed and the efficacy of vaccination for reducing cecal colonization after experimental challenge was evaluated by determining colony-forming units (CFU) of $C$. jejuni in cecal contents.

Results: The CPSconj vaccine was immunogenic when administered as three doses at 3, 4 and 5 weeks of age to specific pathogen free chicks lacking maternal antibodies (seroconversion rates up to $75 \%$ ). Commercial broiler chicks (having maternal antibodies) receiving two doses of CPSconj vaccine at 7 and 21 days of age did not seroconvert before oral challenge at 29 days, but 33\% seroconverted post challenge; none of the placebo-injected, challenged birds seroconverted. Vaccinated birds had significantly lower numbers of C. jejuni in cecal contents than control birds at necropsy (38 days of age). CFU of C. jejuni did not differ significantly among groups of birds receiving CPSconj vaccine with different adjuvants. In two trials, the mean reduction in CFU associated with vaccination was $0.64 \log _{10}$ units.

Conclusions: The CPSconj vaccine was immunogenic in chicks lacking maternal antibodies, vaccinated beginning at 3 weeks of age. In commercial broiler birds (possessing maternal antibodies) vaccinated at 7 and 21 days of age, 33\% of birds seroconverted by 9 days after challenge, and there was a modest, but significant, reduction in cecal counts of C. jejuni. Further studies are needed to optimize adjuvant, route of delivery and scheduling of administration of this vaccine.
\end{abstract}

Keywords: Campylobacter jejuni, Cecum, Capsular polysaccharide, Conjugate vaccine, Vaccination, Broiler chickens

\section{Background}

Food-borne illness due to infection with Campylobacter species has been estimated to cost 1.7 billion dollars a year in medical costs, lost productivity and "qualityadjusted life years" in the United States alone [1]. Reports

\footnotetext{
*Correspondence: shayan@uoguelph.ca

${ }^{\dagger}$ Douglas C Hodgins and Neda Barjesteh contributed equally to the work

1 Department of Pathobiology, University of Guelph, Guelph,

ON N1G 2W1, Canada

Full list of author information is available at the end of the article
}

compiled by the European Food Safety Authority demonstrate increasing numbers of cases in humans over the most recent 4 years of study, in contrast to a steady decrease in the incidence of food-borne Salmonella infections [2]. Contaminated chicken meat is considered the most important source of infection with Campylobacter in developed countries [3]. Broiler chickens typically become infected with Campylobacter jejuni after 3 weeks of age and can harbor $10^{8}$ colony-forming units (CFU) or more per gram of cecal contents [4] 
by slaughter age (5-6 weeks of age). In contrast to the intense diarrhea and vomiting, and severe inflammation of intestinal tissues associated with $C$. jejuni infection in humans [5], chickens do not exhibit signs of clinical illness after colonization by $C$. jejuni, and inflammation of intestinal tissue is not evident histologically [6].

Enhanced biosecurity in poultry flocks and improved hygiene during processing of poultry products have potential to reduce contamination of meat at the retail level, but vaccination of broiler chickens will be needed in conjunction with these approaches to have a major impact on campylobacteriosis in humans [7]. At present there are no licensed vaccines for reduction of colonization of chickens by $C$. jejuni [8]. Various vaccine approaches have been explored in experimental studies in chickens (reviewed by de Zoete et al. [9]), including bacterins [10,11], subunit vaccines [11], live Salmonellavectored vaccines [12-14], and encapsulated particle vaccines $[8,15]$, by parenteral, oral, and nasal routes. Putative virulence factors and potential vaccine antigens have included outer membrane proteins [8], flagellin [11, 16], and transport proteins [12-14].

Recent studies have investigated the role of the capsular polysaccharide of $C$. jejuni in virulence in some species, and its potential as a vaccine antigen [17-20]. The capsular polysaccharide of $C$. jejuni 81-176 has been shown to mediate adherence and invasion of a human embryonic epithelial cell line, and to play a role in induction of diarrhea in a ferret model [21]. Wong et al. [22] have reported that modifications of the structure of the capsule of $C$. jejuni NCTC 11168 are associated with significant impairment of cecal colonization of young chicks. Capsular polysaccharide conjugated to the diphtheria toxoid "crossreacting material 197" $\left(\mathrm{CRM}_{197}\right)$ has been reported to be immunogenic in Aotus monkeys, and to protect against clinical diarrhea, but not colonization, following experimental challenge [17]. Although purified capsular polysaccharides can induce protection against encapsulated bacteria, as T-independent antigens they typically are not immunogenic in young infants or chicks [23, 24], and IgG and memory responses are limited [25]. Conjugation of purified capsular polysaccharide to a protein carrier such as $\mathrm{CRM}_{197}$ induces T-dependent responses, and facilitates antibody responses at an earlier age, with isotype switching to IgG and induction of B cell memory [26].

Although vaccination of broiler chicks is an attractive approach to control colonization, there are immunological and logistical barriers that must be overcome. Immune function is limited in the first 2 weeks posthatch $[27,28]$ and maternal antibodies to $C$. jejuni are common in the sera of young chicks [29]. In addition there is little time for induction of immunity, since broiler birds reach slaughter weight by $5-6$ weeks of age.
In the current studies the immunogenicity of the capsular polysaccharide of $C$. jejuni conjugated to $\mathrm{CRM}_{197}$ was assessed by vaccinating specific pathogen free (SPF) chicks (lacking maternal antibodies), starting at 3 weeks of age (when immune function has matured) and following serum antibody responses. In contrast, the protective efficacy of this antigen for reducing cecal colonization of broiler chicks was assessed by vaccinating commercial broiler chicks (having maternal antibodies) with two doses of vaccine (at 1 and 3 weeks of age), followed by experimental challenge at 4 weeks of age. Thus immunogenicity was tested under conditions favorable to induction of antibody responses, but protective efficacy was tested in commercial chicks using earlier vaccination, consistent with the need to induce immunity as young as possible.

\section{Methods}

\section{Vaccine}

Capsular polysaccharide of C. jejuni strain 81-176 was purified and conjugated to diphtheria toxoid $\mathrm{CRM}_{197}$ to form capsular polysaccharide conjugate (CPSconj) as described previously [17].

\section{Immunogenicity in the absence of maternal antibodies}

Specific pathogen free chicks (SPF, N2 strain, Cornell University Center for Animal Resources and Education, Cornell, New York, USA, 5 chicks per experimental group), lacking detectable maternal antibodies at the time of vaccination, were raised in the isolation facilities of the Ontario Veterinary College and vaccinated subcutaneously at 3 and 4 weeks of age with $10 \mu \mathrm{g}$ CPSconj with either $20 \mu \mathrm{g}$ Quil A (Brenntag Biosector, Frederickssund, Denmark) or $10 \mu \mathrm{g}$ CpG oligodeoxynucleotide (CpG ODN) 2007 (Eurofins Operon, Huntsville, AL, USA) as adjuvants, or received $20 \mu \mathrm{g}$ CPSconj with $20 \mu \mathrm{g}$ Quil A at 3, 4 and 5 weeks of age. Vaccination of these chicks thus occurred under favorable conditions (absence of maternal antibodies) beginning at an age when immune function is considered to be mature [27, 28]. Control (placebo) birds received phosphate buffered saline (PBS) in place of vaccine.

\section{Vaccination and challenge of commercial broiler chicks}

One-day-old female commercial broiler chicks (Ross 308 chicks from Stratford Chick Hatchery, Stratford, ON, Canada) were housed in isolation facilities at the Ontario Veterinary College. Chicks were allocated (8 chicks per group) to receive $25 \mu \mathrm{g}$ CPSconj with $10 \mu \mathrm{g}$ CpG or $100 \mu$ Addavax $^{\mathrm{Tm}}$ (squalene based adjuvant, InvivoGen, San Diego, CA, USA) as adjuvant, or $25 \mu \mathrm{g}$ CPSconj without adjuvant, or PBS as a placebo by subcutaneous injection in a $200 \mu \mathrm{l}$ volume at 7 days post-hatch. Birds 
received a booster dose of $10 \mu \mathrm{g}$ of the same antigen preparation as previously, or PBS at 21 days post-hatch. In contrast to the chicks used in the immunogenicity experiment above, these broiler chicks were obtained from a commercial source and had detectable serum (maternal) antibodies at the time of primary vaccination. At 29 days post-hatch birds were challenged orally with $2 \times 10^{7}$ CFU of $C$. jejuni strain 81-176 prepared by the method of Davis and DiRita [30] (see below). Birds were necropsied at 9 days post-challenge (38 days post-hatch) and dilutions of the cecal contents were plated onto Mueller-Hinton agar containing Preston Campylobacter Selective Supplement (Oxoid, Basingstoke, Hampshire, UK) and incubated at $42^{\circ} \mathrm{C}$ for $48 \mathrm{~h}$ in a microaerobic environment to quantitate $\mathrm{CFU}$ of $C$. jejuni. CFU per gram of cecal contents were calculated based on plate counts, adjusting for dilutions. An additional eight birds (serving as negative controls for challenge) were housed in a separate isolation room, were not vaccinated and were not challenged, but were necropsied at the same time as challenged birds. Blood was collected at 7, 21, 29, 34 and 38 days post-hatch for serological testing. The experiment was repeated a second time using chicks from the same source, following the identical protocol in the same facilities, and the results were pooled for statistical analysis.

\section{Animal ethics}

All experiments were approved by the Animal Care Committee of the University of Guelph (Animal Utilization Protocol number 10R086-1836) and followed the guidelines of the Canadian Council on Animal Care.

\section{Enzyme-linked immunosorbent assay (ELISA) for serum IgG antibodies}

Purified capsular polysaccharide of $C$. jejuni diluted to $40 \mu \mathrm{g} / \mathrm{ml}$ in PBS buffer was coated onto Nunc 96 well $\left(\right.$ MicroWell $^{\mathrm{Tw}}$ untreated polystyrene, Thermo Fisher Scientific, Rochester, New York, USA) plates, $100 \mu \mathrm{l} /$ well, at $37^{\circ} \mathrm{C}$ for $3 \mathrm{~h}$. Plates were washed four times with wash buffer consisting of PBS with $0.5 \%$ fish skin gelatin (Sigma, St. Louis, MO, USA) and 0.05\% Tween 20 (Sigma). Sera were diluted $1 / 20$ in wash buffer and $100 \mu l$ was added to wells in duplicate, followed by a $2 \mathrm{~h}$ incubation at $37^{\circ} \mathrm{C}$. After washing four times, bound antibodies were detected using rabbit anti-chicken IgG (Fc specific) horse radish peroxidase conjugate (Jackson ImmunoResearch Laboratories, West Grove, PA, USA) diluted $1 / 350$, incubating at $37^{\circ} \mathrm{C}$ for $1 \mathrm{~h}$. After washing four times, ABTS (2,2'-azino-di (3-ethyl-benzthiazoline6-sulfonate)) substrate (Kirkegaard and Perry Laboratories, Gaithersburg, Maryland, USA) was added, $100 \mu \mathrm{l} /$ well; $1 \%$ sodium dodecylsulfate (Bio-Rad, Hercules, CA,
USA) was added as stop solution after $1 \mathrm{~h}$ and the optical densities were evaluated at $405 \mathrm{~nm}$. A dilution series of a positive control serum consisting of pooled high titre sera from mature hens was run on each plate. Titres were estimated using the method of Sacks et al. [31]. The limit of detection of the assay was $3.32 \log _{2}$ units.

\section{Bacterial culture}

Campylobacter jejuni strain 81-176 was cultured according to the method of Davis and DiRita [30] to provide bacteria for experimental oral challenge. Briefly, a $10-\mu \mathrm{L}$ loop of frozen $C$. jejuni culture maintained at $-80^{\circ} \mathrm{C}$, was inoculated onto Mueller-Hinton agar (Oxoid) and incubated for $18 \mathrm{~h}$ in a sealed jar using gas packs (CampyGen, Oxoid) to maintain microaerobic conditions. Subsequently, several colonies of $C$. jejuni were inoculated into $100 \mathrm{~mL}$ fresh Mueller-Hinton broth and incubated at $41^{\circ} \mathrm{C}$ in microaerobic conditions for $40 \mathrm{~h}$. The broth culture was centrifuged at $3,500 \times g$ for $10 \mathrm{~min}$ and the bacteria were diluted with PBS (pH 7.4) to attain an optical density corresponding to approximately $4.0 \times 10^{7} \mathrm{CFU} / \mathrm{ml}$, based on previous analysis of growth curves. The number of viable C. jejuni received by the chickens at the time of challenge was established retrospectively by plating dilutions of the inocula onto Mueller-Hinton agar plates.

\section{Statistical analysis}

Data from the two challenge trials were pooled. CFU/ gm of $C$. jejuni in cecal contents were $\log _{10}$ transformed before analysis using a mixed model (Proc Mixed) including trial as a random variable, in SAS version 9.2. Vaccine groups were subsequently compared using Tukey's test. Antibody titres were expressed on a $\log _{2}$ scale. Titres were not normally distributed for the majority of time points; a nonparametric test (Proc Npar1way [KruskalWallis test]) was used to compare antibody titres among groups. Seroconversion (increase in titre by $\geq 2 \log _{2}$ units) rates were compared using Fisher's exact test. Correlation between serum antibody titres on the day of necropsy and CFU of $C$. jejuni in cecal contents was evaluated using Pearson's correlation coefficient using Proc Corr in SAS.

\section{Results and discussion}

Immunogenicity of the CPS conjugate vaccine in the absence of maternal antibodies

Mean serum IgG antibody titres increased in vaccinated SPF birds, but not in negative control birds that received PBS (Figure 1). Seroconversion rates were 20, 40 and 75\% by 6 weeks of age in birds receiving two doses of CPSconj vaccine with $\mathrm{CpG}$, two doses CPSconj with Quil A and three doses CPSconj with Quil A respectively. The seroconversion rate in birds receiving three doses of CPSconj 


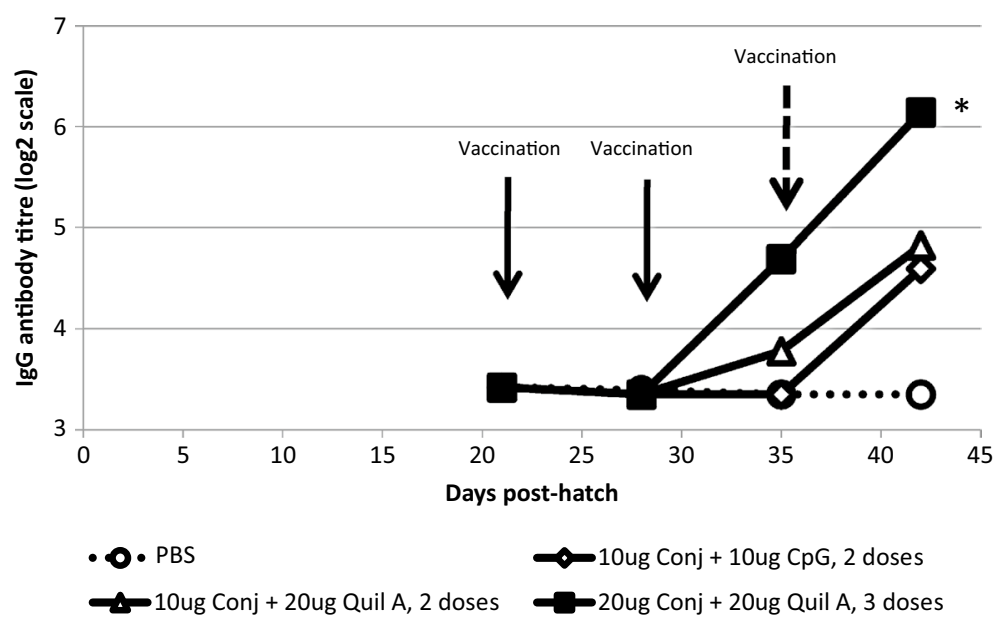

Seroconversion

rate by day 42

$3 / 4 *$

$2 / 5$

$1 / 5$

$0 / 5$

1 Immunogenicity of the capsular polysaccharide-diphtheria toxoid ( $\mathrm{CRM}_{197}$ ) conjugate vaccine. Specific pathogen free chicks (N2 strain), lacking maternal antibodies at the time of primary vaccination, were vaccinated subcutaneously at 3 and 4 weeks post-hatch ( $10 \mu \mathrm{g}$ of conjugate with $10 \mu \mathrm{g} \mathrm{CpG}$ or with $20 \mu \mathrm{g}$ Quil A), or at 3, 4 and 5 weeks post-hatch ( $20 \mu \mathrm{g}$ of conjugate with $20 \mu \mathrm{g}$ Quil A) or received phosphate buffered saline (PBS) as a placebo. Mean serum IgG antibodies specific for capsular polysaccharide of C. jejuni strain 81-176 were assayed by ELISA and titres were expressed on a $\log _{2}$ scale. The limit of detection was $3.32\left(\log _{2}\right.$ scale). Seroconversion [fourfold or greater increase in antibody titre ( $\geq 2 \log _{2}$ units)] rates after vaccination are indicated on the right of the figure for each group. * Seroconversion rate was significantly higher than in birds receiving PBS as placebo ( $p<0.05$, one-tailed Fisher's exact test).

was significantly higher than the rate in birds receiving PBS as placebo $(\mathrm{p}<0.05$, one-tailed Fisher's exact test).

\section{Antibody responses of commercial broiler chicks}

Maternal IgG antibodies were evident in serum at 7 days post-hatch at the time of primary vaccination, but declined considerably by day 21 (day of secondary vaccination, Figure 2). Maternal antibodies have been shown to mediate partial protection against colonization by $C$. jejuni [32], but the short half-life of maternal antibodies (about 5 days in young chicks [33]) suggests that titres of maternal antibodies would need to be considerably higher to provide protection up to slaughter age. Mean serum IgG antibody titres declined further by day of challenge (day 29) in all groups. IgG antibody titres did not differ significantly among the four experimental groups (three groups of vaccinates and one placebo group) at any of the time points (Kruskal-Wallis test). Data from the vaccinated groups (CPS conjugate without adjuvant, CPS conjugate with Addavax ${ }^{\mathrm{Tm}}$ adjuvant and CPS conjugate with $\mathrm{CpG}$ as adjuvant) were pooled for further analysis since there were no significant differences among them.

In Figure 3 the distribution of serum IgG antibody titres in vaccinated broiler birds and birds receiving placebo, is contrasted by means of histograms. Seroconversion (an increase of titre $\geq 2 \log _{2}$ units) was not detected before day of challenge in vaccinates. This is in contrast to responses seen in SPF birds by 3 weeks post primary vaccination (Figure 1). Seroconversion occurred in $33 \%$ of

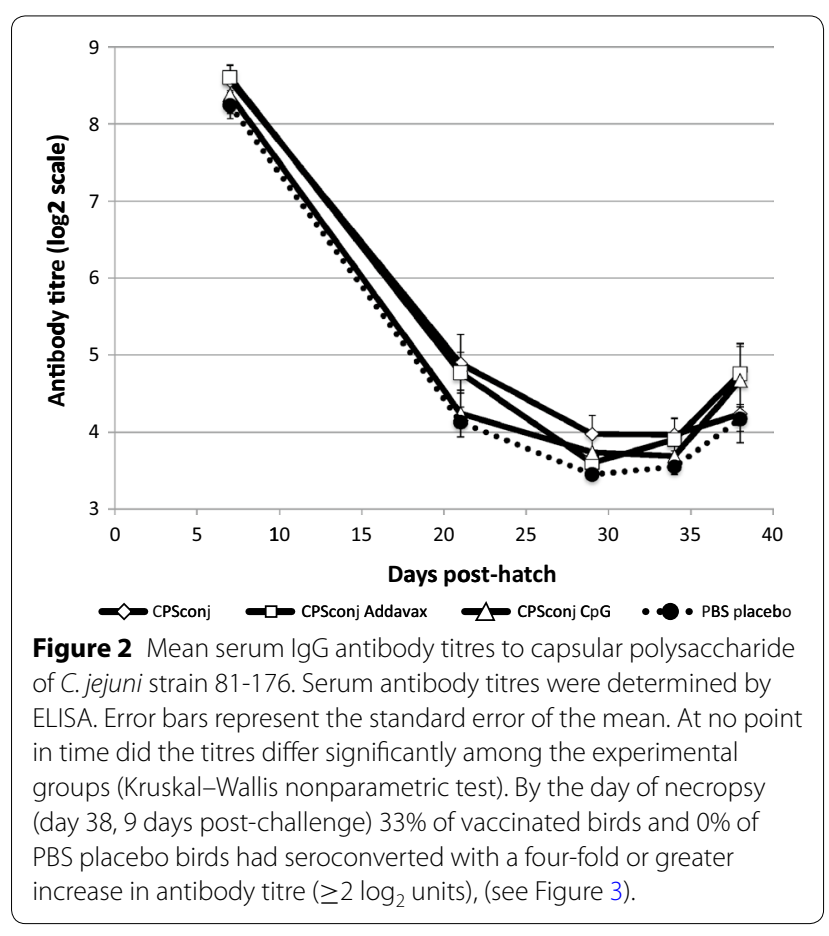

vaccinated broiler birds by 9 days post-challenge, but in none of the birds that had received placebo before challenge ( $\mathrm{p}<0.01$, Fisher's exact two-tailed test). This suggests that although vaccination with two doses of vaccine did not induce increases in circulating antibody titres 


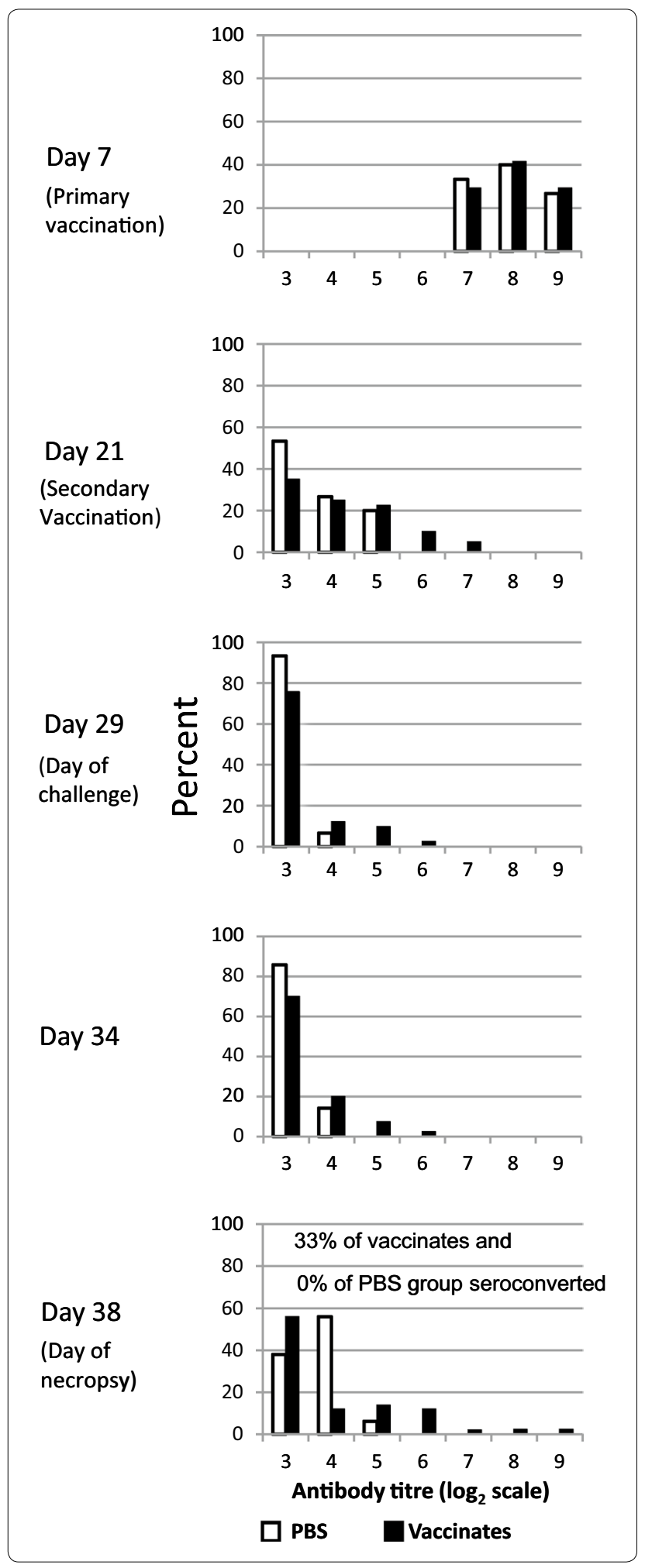

before the day of challenge, there was a degree of priming for anamnestic antibody responses (which occurred after exposure to live $C$. jejuni) in some of the birds. In
Figure 3 Histograms showing distribution of serum lgG antibodies specific for capsular polysaccharide of C. jejuni strain 81-176 in commercial broiler chicks. Chicks were vaccinated at 7 and 21 days posthatch with capsular polysaccharide-conjugate (with CpG ODN 2007, or Addavax $^{\mathrm{TM}}$ as adjuvant, or no adjuvant; antibody titres for the three vaccinated groups were pooled to graph the histograms since they did not differ significantly at any of the time points) or received PBS placebo. IgG antibody titres were determined by ELISA and analyzed using the Kruskal-Wallis (nonparametric) test because at the majority of time points, the titres were not normally distributed. In each panel, the percentage (Y-axis) of (vaccinated and PBS control) birds with particular antibody titres is plotted against their titres at that time point. A shift (to the left) in the distribution of antibody titres, consistent with decline in titres of maternal antibodies, is evident from day 7 posthatch to day 29 (day of challenge), followed by a shift to the right after challenge. Antibody titres did not differ significantly among the four treatment groups at any of the time points. Seroconversion, a fourfold or greater ( $\geq 2 \log _{2}$ units) increase in antibody titre, did not occur in any of the birds before the day of challenge (day 29 post-hatch), but $33 \%$ of vaccinated and $0 \%$ of placebo (PBS) birds seroconverted by day 38 ( 9 days post-challenge, a significant difference, Fisher's exact test $p<0.01$ ). For each time point, sera from 14 to 16 PBS (placebo) birds and sera from 40 to 43 vaccinated birds were assayed.

a similar fashion, Siegrist et al. [34] have reported an absence of serum antibody responses to vaccination in neonatal mice with circulating maternal antibodies. In these mouse experiments anamnestic antibody responses became evident after subsequent antigen exposure.

It is possible that if cecal CFU were monitored beyond 9 days post-challenge, that mean CFU would decline further as immune responses aided in clearance of $C$. jejuni. A gradual decline in cecal colonization in birds vaccinated with live attenuated Salmonella expressing the CjaA protein of $C$. jejuni has been reported by Buckley et al. [14]. Any decline after 5-6 weeks of age, however, is not relevant since most broiler birds are slaughtered by that age. Clearly further research will be needed to improve the immunogenicity of the vaccine for young broilers.

The lower seroconversion rates noted in commercial broiler chicks compared to SPF N strain birds are most probably due to the younger age at primary vaccination of the broilers, or to suppressive effects of residual maternal antibodies, but the effect of different genetic backgrounds cannot be eliminated as a factor in the current study.

\section{Reduction in C. jejuni in cecal contents of vaccinated chicks}

Colony-forming units of $C$. jejuni were significantly lower in the cecal contents of birds receiving CPSconj vaccine (with or without adjuvant) compared to control birds receiving PBS (Table 1). Mean CFU of $C$. jejuni did not differ significantly among groups of vaccinated birds and 
Table 1 Colony-forming units of $C$. jejuni per gram of cecal contents 9 days post-challenge

\begin{tabular}{|c|c|c|c|c|}
\hline Vaccine group & $\mathrm{n}$ & $\begin{array}{l}\text { CFU per gram of cecal } \\
\text { contents (log } \log _{10} \text { transformed) }\end{array}$ & $\begin{array}{l}\text { Standard error } \\
\text { of the mean }\end{array}$ & $\begin{array}{l}p \text { value for comparison } \\
\text { with the PBS group }\end{array}$ \\
\hline PBS & 16 & 8.11 & 0.15 & \\
\hline Conjugate alone & 13 & 7.38 & 0.16 & 0.01 \\
\hline Conjugate $+\mathrm{CpG}$ & 14 & 7.55 & 0.15 & $<0.05$ \\
\hline Conjugate + Addavax ${ }^{\top M}$ & 16 & 7.47 & 0.14 & 0.01 \\
\hline All vaccinates (data pooled) & 43 & 7.47 & 0.09 & $<0.001$ \\
\hline
\end{tabular}

Combined results from two experiments using Ross 308 broiler chicks from the same source and following the same experimental protocol. Chickens were vaccinated subcutaneously at 7 and 21 days post-hatch with capsular polysaccharide conjugate vaccine with or without CpG or Addavax ${ }^{\mathrm{TM}}$ as adjuvant, or received PBS as a placebo. Birds were challenged at 29 days post-hatch and necropsied at 38 days post-hatch.

were pooled for further analysis. Vaccinated birds had a mean reduction in cecal C. jejuni of $0.64 \log _{10}$ units compared to birds receiving PBS as placebo $(\mathrm{p}<0.001)$. There was essentially no correlation between serum antibody titres and cecal counts of $C$. jejuni at necropsy ( $\mathrm{r}$ value of -0.06 among vaccinates with $\mathrm{p}=0.70$, data not shown). Since Campylobacter colonize the mucus layer of the intestinal tract and are rarely found in extra-intestinal sites, a low correlation between serum antibody titres and protection could be anticipated. Assay of antibodies in intestinal mucus should be more informative. Vaccination with CPSconj by a mucosal route might improve vaccine efficacy compared to the subcutaneous route used in the present study, by inducing IgA antibodies at sites of colonization by $C$. jejuni. The subcutaneous route was used in the present study to assess immunogenicity of the vaccine construct and to provide a baseline for comparison as systems for delivery to mucosal sites are developed. Rosenquist et al. [35] have suggested, based on mathematical modeling, that a $2.0 \log _{10}$ unit reduction in $C$. jejuni in chickens at slaughter would reduce clinical illness in humans 30-fold. Buckley et al. [14] have reported reductions in cecal colonization of 1.57-1.91 $\log _{10}$ CFU of C. jejuni per gram of cecal contents in SPF chickens (lacking maternal antibodies) vaccinated with recombinant CjaA protein, but reductions were not evident until 7 weeks of age. Widders et al. [11] have reported reductions of $1.9 \log _{10}$ CFU per gram of cecal contents by 6 weeks of age in birds vaccinated intraperitoneally with antigens of killed $C$. jejuni. Larger reductions in cecal counts of $C$. jejuni ( $6 \log _{10}$ CFU per gram) have been reported with Salmonella vectored vaccines [12], but there are safety issues associated with persistence of the Salmonella vector in vaccinated birds [14].

A previous study vaccinating mature monkeys (lacking pre-existing antibody titres) with a CPSconj vaccine has demonstrated efficacy in reducing clinical signs (in primates), but quantitative studies of intestinal carriage of $C$. jejuni were not carried out [17]. Because $C$. jejuni causes clinical disease in primates, the end goal of vaccination in such studies is reduction in signs of disease. In contrast the goal in vaccinating chickens is to reduce the number of $C$. jejuni in the intestinal tract of the birds at the time of slaughter. This may require a qualitatively different or quantitatively greater immune response and will be a more elusive goal.

\section{Alternative vaccination approaches}

The current study assessed immunogenicity of the capsular polysaccharide antigen conjugated to diphtheria toxoid $\mathrm{CRM}_{197}$. This carrier protein has been used in vaccines for human infants and has been shown to enhance immune responses to polysaccharide antigens in the first months of life [26]. Other carrier proteins have been used in conjugate vaccines [36] and perhaps these or even $C$. jejuni surface proteins may also prove efficacious in chickens. Schijns et al. [37] have reported that some adjuvants enhance the immunogenicity of tetanus toxoid in immunologically mature chickens (3 weeks-old) but fail to induce antibodies to this antigen in day-old chicks, whereas other adjuvants are effective at both ages. Thus it may be necessary to optimize both carrier protein and adjuvant to produce conjugate vaccines immunogenic for day-old chicks.

In the present study vaccines were administered subcutaneously to evaluate immunogenicity of the capsular antigen. Mucosal vaccination, especially by the oral route may be more effective to induce intestinal immune responses and reduce intestinal colonization, but improved adjuvants and delivery systems will be required to make this approach practical.

Further studies will be needed to optimize adjuvants, route of delivery, and scheduling of administration for use of CPSconj antigen in broiler chickens. Studies using heterologous challenge strains of $C$. jejuni are also in order.

\section{Conclusions}

A capsular polysaccharide-diphtheria toxoid conjugate vaccine administered subcutaneously, starting at 3 weeks 
of age, induced serum IgG antibodies in SPF chicks lacking detectable maternal antibodies. Vaccination of broiler chicks (that had maternal antibodies) starting at 7 days post-hatch was not associated with seroconversion with IgG antibodies before challenge at 29 days post-hatch. Some (33\%) of the vaccinated broiler birds seroconverted by 9 days post-challenge, but none of the PBS (placebo) birds, indicating that the vaccine primed for an earlier IgG antibody response following challenge. There was a modest $\left(0.64 \log _{10}\right.$ colony forming units) but significant $(\mathrm{p}<0.001)$ reduction in $C$. jejuni in cecal contents, 9 days post-challenge, in vaccinated birds compared to negative control (placebo) birds. Further studies are needed to optimize adjuvant, route of delivery and scheduling of administration to make the most effective use of this vaccine antigen.

\author{
Abbreviations \\ CFU: colony forming units; PBS: phosphate buffered saline; ODN: oligonu- \\ cleotide; CPSconj: capsular polysaccharide-diphtheria toxoid conjugate; SPF: \\ specific pathogen free.
}

\begin{abstract}
Authors' contributions
DCH and NB contributed equally in the design, and execution of the work. DCH drafted the manuscript. MSP assisted with experimental procedures. ZM and MAM were responsible for the production of the capsular polysaccharideconjugate vaccine, and with the revision of the manuscript. SS contributed to the design of the experiment, the analysis of results and revision of the manuscript. All authors read and approved the final manuscript.
\end{abstract}

\section{Author details}

${ }^{1}$ Department of Pathobiology, University of Guelph, Guelph, ON N1G 2W1, Canada. ${ }^{2}$ Department of Chemistry, University of Guelph, Guelph, Canada. ${ }^{3}$ Department of Immunology, University of Toronto, Toronto, ON, Canada.

\section{Acknowledgments}

Research funding was provided by the Canadian Poultry Research Council, Poultry Industry Council and the Ontario Ministry of Agriculture and Food (SS), and by the Natural Sciences and Engineering Research Council of Canada (SS and MAM). The funding agencies were not involved in the study design, or the collection, analysis, or interpretation of the data, or in the writing of the manuscript, or in the decision to submit the manuscript for publication. The assistance of Dr Shahriar Orouji in immunogenicity testing and the assistance of the staff of the isolation unit of the Ontario Veterinary College, are gratefully acknowledged.

\section{Compliance with ethical guidelines}

\section{Competing interests}

The authors declare that they have no competing interests.

Received: 11 February 2015 Accepted: 20 May 2015

Published online: 02 June 2015

\section{References}

1. Hoffmann S, Batz MB, Morris JG (2012) Annual cost of illness and quality-adjusted life year losses in the United States due to 14 foodborne pathogens. J Food Prot 75:1292-1302

2. European Food Safety Authority/European Centre for Disease Control (2013) The European Union summary report on trends and sources of zoonoses, zoonotic agents and food-borne outbreaks in 2011. EFSA J 11:3129
3. Young KT, Davis LM, DiRita VJ (2007) Campylobacter jejuni: molecular biology and pathogenesis. Nat Rev Microbiol 5:665-679

4. Sahin O, Morishita TY, Zhang Q (2002) Campylobacter colonization in poultry: sources of infection and means of transmission. Anim Health Res Rev 3:95-105

5. LiY-P, Ingmer H, Madsen M, Bang DD (2008) Cytokine responses in primary chicken embryo intestinal cells infected with Campylobacter jejuni strains of human and chicken origin and the expression of bacterial virulence-associated genes. BMC Microbiol 8:107

6. Dhillon AS, Shivaprasad HL, Schaberg D, Wier F, Weber S, Bandli D (2006) Campylobacter jejuni infection in chickens. Avian Dis 50:55-58

7. Newell DG, Elvers KT, Dopfer D, Hansson I, Jones P, James S et al (2011) Biosecurity-based interventions and strategies to reduce Campylobacter spp. on poultry farms. Appl Environ Microbiol 77:8605-8614

8. Annamalai T, Pina-Mimbela R, Kumar A, Binjawadagi B, Liu Z, Renukaradhya GJ et al (2013) Evaluation of nanoparticle-encapsulated outer membrane proteins for the control of Campylobacter jejuni colonization in chickens. Poult Sci 92:2201-2211

9. de Zoete MR, van Putten JPM, Wagenaar JA (2007) Vaccination of chickens against Campylobacter. Vaccine 25:5548-5557

10. Burr DH, Rollins D, Lee LH, Pattarini DL, Walz SS, Tian JH et al (2005) Prevention of disease in ferrets fed an inactivated whole cell Campylobacter jejuni vaccine. Vaccine 23:4315-4321

11. Widders PR, Perry R, Muir WI, Husband AJ, Long KA (1996) Immunisation of chickens to reduce intestinal colonization with Campylobacter jejuni. Br Poult Sci 37:765-778

12. Wyszynska A, Raczko A, Lis M, Jagusztyn-Krynicka EK (2004) Oral immunization of chickens with avirulent Salmonella vaccine strain carrying C. jejuni 72Dz/92 cjaA gene elicits specific humoral immune response associated with protection against challenge with wild-type Campylobacter. Vaccine 22:1379-1389

13. Layton SL, Morgan MJ, Cole K, Kwon YM, Donoghue DJ, Hargis BM et al (2011) Evaluation of Salmonella-vectored Campylobacter peptide epitopes for reduction of Campylobacter jejuni in broiler chickens. Clin Vaccine Immunol 18:449-454

14. Buckley AM, Wang J, Hudson DL, Grant AJ, Jones MA, Maskell DJ et al (2010) Evaluation of live-attenuated Salmonella vaccines expressing Campylobacter antigens for control of C. jejuni in poultry. Vaccine 28:1094-1105

15. Huang JL, Yin YX, Pan ZM, Zhang G, Zhu AP, Liu XF et al (2010) Intranasal immunization with chitosan/pCAGGS-flaA nanoparticles inhibits Campylobacter jejuni in a White Leghorn model. J Biomed Biotechnol. doi:10.1155/2010/589476

16. Khoury CA, Meinersmann RF (1995) A genetic hybrid of the Campylobacter jejuni flaA gene with LT-B of Escherichia coli and assessment of the efficacy of the hybrid protein as an oral chicken vaccine. Avian Dis 39:812-820

17. Monteiro MA, Baqar S, Hall ER, Chen Y-H, Porter CK, Bentzel DE et al (2009) Capsule polysaccharide conjugate vaccine against diarrheal disease caused by Campylobacter jejuni. Infect Immun 77:1128-1136

18. Guerry P, Poly F, Riddle M, Maue AC, Chen YH, Monteiro MA (2012) Campylobacter polysaccharide capsules: virulence and vaccines. Front Cell Infect Microbiol 2:7

19. Bertolo L, Ewing CP, Maue A, Poly F, Guerry P, Monteiro MA (2013) The design of a capsule polysaccharide conjugate vaccine against Campylobacter jejuni serotype HS15. Carbohydr Res 366:45-49

20. Maue AC, Mohawk KL, Giles DK, Poly F, Ewing CP, Jiao Y et al (2013) The polysaccharide capsule of Campylobacter jejuni modulates the host immune response. Infect Immun 81:665-672

21. Bacon DJ, Szymanski CM, Burr DH, Silver RP, Alm RA, Guerry P (2001) A phase-variable capsule is involved in virulence of Campylobacter jejuni 81-176. Mol Microbiol 40:769-777

22. Wong A, Lange D, Houle S, Arbatsky NP, Valvano MA, Knirel YA et al (2015) Role of capsular modified heptose in the virulence of Campylobacter jejuni. Mol Microbiol. doi:10.1111/mmi.12995

23. Jeurissen SHM, Janse EM, van Rooijen N, Claassen E (1998) Inadequate anti-polysaccharide antibody responses in the chicken. Immunobiology 198:385-395

24. Murphy K (2012) Janeway's immunobiology, 8th edn. Garland Science, London, $\mathrm{p} 407$ 
25. Tizard IR (2013) Veterinary immunology, 9th edn. Elsevier Saunders, St. Louis, p 156

26. Klein Klouwenberg P, Bont L (2008) Neonatal and infantile immune responses to encapsulated bacteria and conjugate vaccines. Clin Dev Immunol. doi:10.1155/2008/628963

27. Mast J, Goddeeris BM (1999) Development of immune competence of broiler chickens. Vet Immunol Immunopathol 70:245-256

28. Bar-Shira E, Sklan D, Friedman A (2003) Establishment of immune competence in the avian GALT during the immediate post-hatch period. Dev Comp Immunol 27:147-157

29. Sahin O, Zhang Q, Meitzler JC, Harr BS, Morishita TY, Mohan R (2001) Prevalence, antigenic specificity, and bactericidal activity of poultry anti-Campylobacter maternal antibodies. Appl Environ Microbiol 67:3951-3957

30. Davis L, DiRita V (2008) Growth and laboratory maintenance of Campylobacter jejuni. Curr Protoc Microbiol, Chapter 8, Unit 8A.1.1-8A.1.7

31. Sacks JM, Gillette KG, Frank GH (1988) Development and evaluation of an enzyme linked immunosorbent assay for bovine antibody to Pasteurella haemolytica: constructing an enzyme linked immunosorbent assay titer. Am J Vet Res 49:38-41
32. Sahin O, Luo N, Huang S, Zhang Q (2003) Effect of Campylobacter-specific maternal antibodies on Campylobacter jejuni colonization in young chickens. Appl Environ Microbiol 69:5372-5379

33. Härtle S, Magor KE, Göbel TW, Davison F, Kaspers B (2014) Structure and evolution of avian immunoglobulins. In: Schat KA, Kaspers B, Kaiser P (eds) Avian immunology, 2nd edn. Elsevier, Amsterdam, p 108

34. Siegrist CA, Barrios C, Martinez X, Brandt C, Berney M, Córdova M et al (1998) Influences of maternal antibodies on vaccine responses: inhibition of antibody but not $\mathrm{T}$ cell responses allows successful early prime-boost strategies in mice. Eur J Immunol 28:4138-4148

35. Rosenquist H, Nielsen NL, Sommer HM, Norrung B, Christensen BB (2003) Quantitative risk assessment of human campylobacteriosis associated with thermophilic Campylobacter species in chickens. Int J Food Microbiol 83:87-103

36. Goldblatt D (2000) Conjugate vaccines. Clin Exp Immunol 119:1-3

37. Schijns VE, Weining KC, Nuijten P, Rijke EO, Staeheli P (2000) Immunoadjuvant activities of $E$. coli- and plasmid-expressed recombinant chicken IFNalpha/beta, IFN-gamma and IL-1 beta in 1-day-and 3-week-old chickens. Vaccine 18:2147-2154

\section{Submit your next manuscript to BioMed Central and take full advantage of:}

- Convenient online submission

- Thorough peer review

- No space constraints or color figure charges

- Immediate publication on acceptance

- Inclusion in PubMed, CAS, Scopus and Google Scholar

- Research which is freely available for redistribution

Submit your manuscript at 